EBM-Vergütungsreform zum 1. Juli

\title{
Nur bei Regelleistungsvolumen sind Euro fest
}

\author{
„Stabile Regelleistungsvolumen (RLV) durch neue Verteilungsregeln" lautet \\ die Devise der Kassenärztlichen Bundesvereinigung (KBV). Man könnte das \\ auch anders ausdrücken: Die Honorar-Reform EBM 200oplus ist gescheitert.
}

- Betriebswirtschaftlich kalkuliert sollte der neue EBM bei einem Punktwert von 5,11 Cent nach über einem Jahrzehnt ständigen Honorarverlustes endlich zu einer akzeptablen Vergütung führen. Der unparteiische Vorsitzende des Schiedsausschusses, Prof. Gitter, hatte 5,11 Cent als betriebswirtschaftlich unterste Grenze anerkannt. Dieses Minimum wurde nie bezahlt.

Ab 1. Juli werden die RLV zu festen Euro-Beträgen bezahlt. Dafür wird der Rest einer starren Budgetierung unterzogen. Hiervon ausgenommen sind im Prinzip nur noch Leistungen, die außerhalb der morbiditätsbezogenen Gesamtvergütung (MGV) liegen.

Der Bewertungsausschuss hat den KVen in Absprache mit den Krankenkassen einige Varianten der QZV-Berechnung vorgegeben:

\section{Seminarankündigung EBM 2010}

Unser Gebührenordnungsexperte Helmut Walbert macht Sie rechtzeitig fit mit Strategien zu qualitätsbezogenen Zusatzvolumen (OZZ), RLV, Budgets:

$\begin{array}{lr}\text { Orte und Termine: } & \\ \text { Köln: } & \text { 9. Juni } 2010 \\ \text { Hannover: } & \text { 30. Juni } 2010 \\ \text { Hamburg: } & \text { 2. Juli } 2010 \\ \text { Berlin: } & \text { 3. Juli } 2010 \\ \text { Nürnberg: } & \text { 7. Juli } 2010 \\ \text { München: } & \text { 10. Juli } 2010\end{array}$

Nähere Infos unter:

Tel.: 09312998594

Fax: 093125090601

E-Mail:w@lbert.info

Website: www.walbert-helmut.de

\section{Berechnung je Leistungsfall}

Es wird neben den bereits bekannten Begriffen „Arztfall“, „Behandlungsfall“ und „RLV-Fall“ eine weitere Definition eingeführt: der „Leistungsfall“. Dieser liegt dann vor, wenn in den Behandlungsfällen des Vorjahresquartals mindestens eine Leistung aus dem Leistungskatalog des entsprechenden QZV abgerechnet wurde. Der für das QZV zur Verfügung stehende Euro-Betrag wird durch die Anzahl der Leistungsfälle des Vorjahresquartals derjenigen Ärzte geteilt, die Anspruch auf dieses QZV haben. Das ergibt den QZV-Fallwert. Bei dieser Variante berechnet sich das QZV-Volumen der einzelnen Praxis durch die Multiplikation des QZVFallwertes mit der Leistungsfall-Zahl.

\section{Berechnung je RLV-Fall}

Der für das QZV zur Verfügung stehende Euro-Betrag wird durch die Zahl der RLV-Fälle des Vorjahresquartals der Ärzte geteilt, die Anspruch auf das entsprechende QZV haben. Dies ergibt den QZV-Fallwert. Durch Multiplikation dieses Wertes mit RLV-Fallzahl ergibt sich das Volumen des QZV für den einzelnen Arzt. Hier wird lediglich die Leistungsbreite einer Praxis berücksichtigt, nicht die eventuell notwendigerweise zu erbringende Leistungsmenge.

\section{Berechnung je Arzt}

Das QZV kann auch arztspezifisch berechnet werden: Als Erstes stellt die KV die Anzahl der Ärzte fest, die einen Anspruch auf das jeweilige QZV haben. Dann wird der für das QZV zur Verfü- gung stehende Euro-Betrag durch die Anzahl der berechtigten Ärzte geteilt. Der sich ergebende Betrag ist dann ein einheitliches QZV für alle berechtigten Ärzte. Diese Berechnung erfasst nur die Leistungsbreite.

Das Ganze erhält noch einen besonderen Touch dadurch, dass die KVen diese Berechnungsmöglichkeiten nicht einheitlich auf alle QZV anwenden müssen. Auswahlkriterium könnte die Art oder/und die Menge der jeweiligen Leistungen sein. So könnte beispielsweise eine KV das QZV „Unvorhergesehene Inanspruchnahme" nach der Variante „3“ je Arzt vergüten. Das QZV "Sonografie“ könnte nach Variante 2 je RLV-Fall berechnet werden und das QZV „Kleine Operationen“ nach Variante „1“ je Leistungsfall.

Damit sind die Möglichkeiten des Vernebelns noch nicht am Ende! Kassen und KVen können sich darauf einigen, ausgewählte QZV zusammenzufassen, einzelne QZV mit dem RLV zusammenzufassen oder weitere QZV zu definieren, die über die vom Gemeinsamen Ausschuss definierten QZV hinausgehen.

Da bei diesen vielfältigen Berechnungsmöglichkeiten die Chancen für Verwerfungen in der einzelnen Vertragsarztpraxis erheblich steigen, kann weiter die sogenannte Konvergenzregelung bis Ende 2011 angewendet werden. Der Beschluss verpflichtet die KVen jedoch, dass die Anpassung bis Ende 2011 in "quartalsweise erfolgenden Schritten“" umgesetzt werden muss. Ab dann ist das Ende des Lernprozesses erreicht: entweder systemimmanent arbeiten oder die entsprechenden Konsequenzen tragen.

H. WALBERT = 